\title{
COMPORTAMENTO DOS CUSTOS TOTAIS DE PRODUÇÃO NO SEGMENTO DA AVICULTURA DE POSTURA NO ESTADO DO PARANÁ: ESTUDO BASEADO NA ANÁLISE DE REGRESSÃO LINEAR MÚLTIPLA
}

\section{BEHAVIOR OF THE TOTAL COSTS OF PRODUCTION IN THE POULTRY INDUSTRY IN THE STATE OF PARANÁ: A STUDY BASED ON THE ANALYSIS OF MULTIPLE LINEAR REGRESSION TÍTULO DO TRABALHO EM PORTUGUÊS}

\author{
Letícia de Oliveira*E-mail: letydeoliveira@hotmail.com \\ Paulo César Tonin* E-mail: pctonin@utfpr.edu.br \\ Silvana Lígia Vicenzi`E-mail: sligie@globo.com \\ *Universidade Tecnológica Federal do Paraná (UTFPR), Curitiba, PR
}

Resumo: O trabalho de pesquisa teve por objetivo determinar uma função matemática, para descrever os padrões de comportamento dos custos totais, em função das variáveis, produtividade, mortalidade, consumo de ração e horas trabalhadas em um aviário manual de produção de ovos. Os dados coletados foram referentes ao período compreendido entre março de 2016 a janeiro de 2017. Após a identificação dos dados, foi aplicada a análise de regressão linear múltipla para verificação do grau de relacionamento entre as variáveis estudadas e, ainda, para a identificação da equação capaz de linearizar o comportamento dos "custos totais" em função do comportamento das variáveis independentes. Para validação das modelagens pesquisadas, além das análises envolvendo o coeficiente de determinação, a estatística $t$ e testes de significância de coeficientes, foram realizados testes estatísticos envolvendo a análise de presença de multicolinearidade, autocorrelação de resíduos e a normalidade de resíduos. A regressão linear múltipla apresentou um coeficiente de determinação de 0,95, indicando que as variáveis "produtividade" e "mortalidade" explicam 95\% das variações nos "custos totais de produção". Diante dos resultados obtidos, foi possível concluir que houve correlação e conseguiu-se determinar uma equação matemática para os padrões de comportamento dos custos totais, em função das variáveis "produtividade" e "mortalidade".

Palavras-chave: Produtividade. Mortalidade. Multicolinearidade.

\begin{abstract}
The objective of this research was to determine a mathematical function to describe behavioral patterns of total costs, as a function of variables, productivity, mortality, feed intake and hours worked in a manual egg production aviary. The data collected were related to the period from March 2016 to January 2017. After the identification of the data, multiple linear regression analysis was applied to verify the degree of relationship between the studied variables and also to identify the equation capable of linearizing the behavior of the "total costs" as a function of the behavior of the independent variables. In order to validate the models studied, in addition to the analyzes involving the coefficient of determination, the t statistic and tests of significance of coefficients, statistical tests were performed involving the analysis of the presence of multicollinearity, autocorrelation of residues and the normality of residues. The multiple linear regression presented a coefficient of determination of 0.95 , indicating that the variables "productivity" and "mortality" account for $95 \%$ of the changes in "total production costs". In view of the results obtained, it was possible to conclude that there was correlation and it was possible to determine a mathematical equation for the behavioral patterns of the total costs, as a function of the variables "productivity" and "mortality".
\end{abstract}

Keywords: Productivity. Mortality. Multicollarity. 


\section{INTRODUÇÃO}

Constituindo-se uma das principais atividades do setor agropecuário do Brasil, a avicultura de postura tem se transformado, ao longo dos anos, em um empreendimento de grande rentabilidade. Em 2016, o ovo passou a ser mais frequente no prato do consumidor brasileiro, devido ao cenário de crise econômica no país (AVISITE, 2017).

Outra justificativa pelo maior consumo de ovos pode ser pelo fato da atividade proporcionar uma das mais importantes fontes de proteína de origem animal. $\mathrm{O}$ ovo é avaliado como um alimento de alto valor nutritivo e com um custo baixo, sendo que qualquer pessoa, não importando a classe social, poderá comprá-lo (VIEIRA, 2015).

Devido a esse crescente desenvolvimento, muitas cooperativas trabalham em conjunto com os avicultores, formando uma cadeia integrada. Onde normalmente os produtores entram com o espaço físico e os investimentos em instalações e a cooperativa integradora fornece as galinhas poedeiras, alimentação, medicamentos, assistência técnica e o transporte.

Diante desses investimentos, os produtores objetivam ter produtividade e lucratividade na produção de ovos. Para que isso aconteça, é fundamental que se faça uma boa gestão de custos. Segundo Melo et al. (2016), uma ótima gestão de custos pode caracterizar um diferencial competitivo para o produtor; capaz de reportar os prováveis prejuízos por falta de planejamento, principalmente na cadeia produtiva de ovos.

A gestão dos custos das atividades desenvolvidas nas propriedades e a apuração dos resultados torna-se um desafio para os produtores rurais. A gestão de custos fornece diversas informações úteis ao produtor, entre elas está à identificação dos custos de produção. Identificar os custos e suas variações é condição fundamental para que a escolha tomada seja a melhor decisão, alcançando assim, resultados otimizados e satisfatórios (SILVA et al., 2007).

Nesse contexto, Medeiros (2005) destaca a importância que os estudos sobre o comportamento dos custos estão se apresentando em vários setores, sendo sua principal finalidade a ação de competitividade. As funções matemáticas são utilizadas para expressar os padrões de comportamentos dos custos, buscando resultados satisfatórios "que expliquem da melhor maneira, como os padrões de 
comportamento de custo variam em função das modificações ocorridas com seus direcionadores" (SILVA et al., 2007, p. 63).

A aplicação da análise de regressão para o cálculo do comportamento dos custos é uma técnica que ajuda o produtor a determinar quão bem a equação explica a relação entre custo e direcionador. O processo de regressão, também, possibilita a inclusão de mais de uma variável explicativa (regressão múltipla) (IUDÍCIBUS, 1989; LEONE, 2000; HORNEGREN; FOSTER; DATAR, 2000; GARRISON; NOREEN, 2001; MAHER, 2001).

Diante desse cenário, espera-se que os avicultores brasileiros busquem ferramentas de apoio à tomada de decisão e que sejam capazes de avaliar e direcionar o seu desempenho econômico e financeiro. Valendo-se das informações de custos totais mensais e de outros dados da produção de ovos, fornecidos pelo avicultor e, mediante a aplicação da metodologia estatística de regressão linear múltipla, o presente trabalho de pesquisa teve por objetivo determinar uma função matemática, que busca relatar os padrões de comportamento dos custos totais, em função das variáveis independentes (produtividade, consumo de ração, mortalidade e horas trabalhadas), em um aviário manual de produção de ovos, localizado na região oeste do estado do Paraná.

\section{REFERENCIAL TEÓRICO}

No referencial teórico, apresentar-se-ão os conceitos considerados necessários ao fundamento do trabalho, tais como a avicultura, a análise de custos, a gestão de custos e a regressão linear múltipla.

\subsection{Avicultura e Análise de Custos}

A avicultura se tornou uma atividade de grande sucesso no agronegócio nacional e internacional, sendo símbolo de crescimento e de modernização. A avicultura de postura, voltada à produção de ovos, tornou-se tanto para os pequenos produtores como para as grandes criações, uma excelente fonte de renda (BIASI et al., 2011). Segundo Helenco e Rigon (2015), a avicultura além de ser desenvolvida em qualquer tipo de propriedade, ainda tem a vantagem de não precisar das 
condições climáticas para o prosseguimento da atividade.

A avicultura é desenvolvida a partir das cooperativas com a participação de avicultores, estes que respondem pelos cuidados e tratos no processo de produção. Os avicultores constroem as instalações e recebem das empresas as aves, a ração e a assistência técnica, tal sistema é conhecido como produção integrada (AZEVEDO et al., 2012).

A produção de ovos no mundo, entre os anos de 2010 a 2015 teve um aumento em 38,7\%. Em 2015, foi produzido quase 1,338 bilhões de ovos em todo o mundo (POULTRY TRENDS, 2015). Segundo o IBGE (2017), a produção de ovos de galinha no ano de 2016 registrou um aumento de 5,8\% em relação ao ano de 2015. A produção anual foi de 3,10 bilhões de dúzias em 2016.

Com o aumento da competitividade e crescentes desafios nos custos de produção para a avicultura, as empresas se vêm forçadas a diferenciar-se com resultados de produtividade e eficiência para sobreviver nesse meio (GALLO, 2015). Crepaldi (2009, p. 277) destaca que "para ser competitivo, o avicultor precisa conhecer seus custos, ampliar a linha de produção e buscar parcerias".

Nos últimos anos, a contabilidade de custos vem evoluindo e modernizandose, tornando-se um importante instrumento de controle e de suporte as tomadas de decisões (CREPALDI, 2010). "A contabilidade de custos é uma técnica utilizada para identificar, mensurar e informar os custos dos produtos e serviços" (CREPALDI, 2009, p. 2). "Os custos podem ser definidos como medidas monetárias dos sacrifícios com os quais uma organização tem que arcar a fim de atingir seus objetivos" (BRUNI; FAMÁ, 2012, p. 5). Segundo Martins (2010, p. 25), "custo é um gasto relativo à bem ou serviço utilizado na produção de outros bens ou serviços".

$\mathrm{Na}$ classificação de custos, o contador determina diferentes sistemas de custos e usam critérios diferentes de avaliação, cálculo e aplicação para fornecer informações específicas para a administração e para os ambientes dos sistemas produtivos (LEONE; LEONE, 2010). A classificação facilita o controle e o processo de tomada de decisão, pois as informações têm diferentes propósitos, usuários e níveis de complexidade (SILVA; LINS, 2013).

Os custos quanto a sua apropriação aos produtos são classificados em custos diretos e custos indiretos. Para Megliorini (2012, p. 9), os custos diretos são 
aqueles "apropriados aos produtos conforme o consumo, exemplos clássicos a matéria-prima e a mão de obra direta".

Os custos indiretos "são custos que complementam uma atividade e são incorridos de forma indireta ou geral, beneficiando, dessa maneira, todos os bens ou serviços produzidos ou serviços prestados" (SANTOS, 2011, p. 25). Em empresas modernas, estes estão se tornando cada vez mais importantes, fazendo com que a discussão sobre a alocação desses custos tenha relevância crescente (BÓRNIA, 2010).

O comportamento dos custos em relação ao volume possibilita a análise das variações nos custos totais e unitários em relação a diferentes volumes de produção. Os custos fixos "em determinado período de tempo e em certa capacidade instalada, não variam, qualquer que seja o volume de atividade da empresa. Existem mesmo que não haja produção" (BRUNI; FAMÁ, 2012, p. 12). Os custos variáveis "são os que variam proporcionalmente ao volume produzido. Exemplo: matéria-prima, embalagem" (CREPALDI, 2010, p. 9).

\subsection{Gestão de Custos}

Na propriedade rural, uma ótima gestão de custos, talvez represente um dos maiores desafios organizacional, principalmente se levarmos em consideração a forte competitividade entre os vários mercados. Assimilar o comportamento dos custos de produção durante uma serie histórica conhecida, pode ajudar o produtor a avaliar o desempenho da atividade exercida na propriedade e auxilia-lo no processo de tomada de decisão (MELO et al., 2016).

A gestão de custos é definida por Martins (2010) como um ramo da contabilidade que tem por objetivo fornecer informações úteis para as empresas, para que as mesmas possam tomar decisões com a finalidade de aumentar a competitividade. Carvalho, Fiúza e Lopes (2008) confirmam essa definição da gestão de custos, em um estudo que realizaram, onde que ao analisar de forma detalhada os custos de produção, puderam concluir que os resultados obtidos, permitiram analisar que a empresa podia permanecer na atividade e ainda se tornar mais competitiva. Sobre uma boa gestão no agronegócio Raíces (2003) aponta: 
Ser um bom produtor rural vai muito além de garantir qualidade, obter boa produtividade ou manter uma criação sadia. A atividade exige 0 conhecimento de todos os custos envolvidos na operação [...]. O sucesso na agricultura, como em qualquer outra empresa, vem da atenção à gestão dos custos de produção, à capacitação do pessoal e à seleção de insumos (RAÍCES, 2003, p. 12 e14).

Com os custos estruturados, o produtor consegue extrair informação que o ajudará na tomada de decisões no decorrer do ciclo produtivo, além de determinar o momento exato para a negociação de sua produção, garantindo assim a rentabilidade de seu negócio (ANDRADE et al., 2012).

Sell (2005) confirma que a decisão pela melhor alternativa, vai definir a ação para atingir os objetivos propostos. Para que o gestor seja prático na seleção da melhor alternativa entre às disponíveis e considerando que seus resultados só serão conhecidos no futuro, é necessário que seja ofertado o suporte necessário de informações e dados que possibilitem a previsão dos resultados alcançados decorrentes da escolha das diversas alternativas. Para tanto, verifica-se aí, a utilidade dos modelos quantitativos para minimização do risco da decisão.

\subsection{Regressão Linear Múltipla}

Nas empresas há, muitas vezes, a necessidade de descrever e prever o comportamento de certas variáveis importantes para a tomada de decisões, tais como: custos, despesas, resultados. As técnicas utilizadas em muitos estudos são a regressão e a correlação; ambas compreendem a análise de dados amostrais para obter informações sobre se duas ou mais variáveis são relacionadas e qual a natureza desse relacionamento (CORRAR et al., 2009).

Maher (2001, p. 405) destaca as técnicas de regressão, como "o procedimento estatístico utilizado para estimar a relação entre variáveis de custos. Essas técnicas determinam a qualidade da função para a estimativa dos custos, ajudando os tomadores de decisões". Segundo Downing e Clark (2006, p. 263), "a regressão linear múltipla permite a inclusão de mais de uma variável explicativa do comportamento dos custos".

Sell (2005) ao observar a aplicabilidade da regressão linear como ferramenta de decisão na gestão de custos, afirma que essa ferramenta estatística é utilizada 
para estimar os valores de uma variável, com base nos valores de outra variável conhecida, explicar os valores de uma variável com base nos valores da outra e prever futuros valores de uma variável.

Levando-se em conta o raciocínio de Hansen e Mowen (2003) e adequando à formulação proposta por Anderson et al. (2007), a Equação 1 representa a regressão linear múltipla como obtenção de uma equação para o custo total:

$C_{T}=F+V_{1} X_{1}+V_{2} X_{2}+\ldots+V_{k} X_{k}$

De forma análoga Maher (2001), destaca que " $C_{T}$ " assume o papel da variável dependente ou, ainda, variável cujo comportamento será estudado. A variável " $F$ " passa a representar o intercepto do eixo das ordenadas e, portanto, 0 montante de custos fixos. As variáveis " $V$ " representam os itens de custo por cada unidade direcionadora escolhida e as variáveis " $X$ " representam as medidas de produção ou de atividade escolhida para o processo de análise mediante a regressão linear múltipla.

Maher (2001), ao abordar o grau de confiabilidade das fórmulas de custos conseguidas mediante o uso da análise de regressão, aponta alguns indicadores a serem analisados com relação à qualidade da equação encontrada, dentre eles, destaca-se o coeficiente de determinação.

Com relação ao coeficiente de determinação, Corrar et al. (2012) destacam que ele é o quadrado do coeficiente de correlação e indica o poder de explicação do modelo em função das variáveis independentes consideradas. Outra análise importante a ser observada é a análise de variância que é um teste de hipóteses, que tem como objetivo constatar a relação entre as variáveis explicativas $\mathrm{Xi}$ e a variável explicada. A metodologia adotada sugere para que sejam enunciadas as hipóteses $H_{0}$ e $H_{1}$, se $H_{0}: \beta_{1}=\beta_{2}=\beta_{3}=\beta_{4}=\beta_{5}=0$ contra a hipótese alternativa $H_{1}$ : existe um $\mathrm{i}$ tal que $\beta_{\mathrm{i}} \neq 0(\mathrm{i}=1,2, \ldots, \mathrm{p})$. Posteriormente adota-se um nível de significância, geralmente em $5 \%$. Caso o valor calculado seja inferior a 0,05 , rejeitase a hipótese $\mathrm{H}_{0}$ e admite-se que há regressão.

Existem procedimentos que são utilizados como processo de tentativa e erro para encontrar as melhores estimativas de regressão. O modelo de eliminação 
backward estima uma equação de regressão utilizando todas as variáveis disponíveis e então vão sendo eliminadas aquelas que não contribuem significativamente com o poder preditivo do modelo (CORRAR et al., 2012, p. 159).

$\mathrm{Na}$ regressão linear múltipla, os testes de significância $t$ e $F$ têm propósitos diferentes, ou seja, o teste $F$ é utilizado para determinar se existe uma relação significativa entre a variável dependente e o conjunto de todas as variáveis independentes. Já o teste $t$ é usado para avaliar se o coeficiente de uma variável independente é valido ou não (ANDERSON et al., 2011)

Ao abordar os pressupostos da modelagem por meio da análise de regressão, Corrar et al. (2012) apontam três problemas que normalmente podem advir do emprego desta técnica estatística: a normalidade dos resíduos, a autocorrelação dos resíduos e a multicolinearidade.

Com relação à verificação da normalidade dos resíduos, ela pode ser efetuada por meio de gráficos ou de testes estatísticos, dentre os quais cabe destacar o Kolmogorov-Smirnov, Shapiro-Wilk e o de Jarque-Bera (CORRAR et al, 2012).

A autocorrelação decorre da influência sobre os resíduos de variáveis independentes importantes, não consideradas na equação. Um modelo sem perturbações deve apresentar resíduos independentes, distribuídos de forma aproximada à distribuição normal. A verificação pode ser realizada graficamente ou através de testes estatísticos como Durbin-Watson e Breusch-godfrey (CORRAR et al, 2012). Segundo Fávero et al. (2009), problemas de autocorrelação dos resíduos podem ser identificados por meio da formulação da estatística $d$ de Dubin-Watson. A estatística $d$ é aproximadamente igual a $2(1-\hat{p})$, sendo que, $\hat{p}$ é coeficiente de correlação entre a amostra e os resíduos. Logo, se a estatística $d$ gerar um valor próximo de dois, não há indícios de autocorrelação entre os resíduos. Por outro lado, ocorrerá a autocorrelação entre os resíduos se dos valores da estatística $d$ forem significantemente diferentes de dois.

Caracteriza-se a multicolinearidade como uma alta correlação entre duas ou mais variáveis independentes em um modelo de regressão linear múltipla. Isto deve ser evitado, pois acarreta em perturbações ao modelo, resultando em valores estimados imprecisos. Esta condição pode ser feita determinando-se os coeficientes 
de correlação simples entre as variáveis independentes. Caso sejam encontrados valores absolutos próximos de 1 (um) na correlação entre qualquer par de variáveis, certamente haverá multicolinearidade. Valores de até 0,40 não sugerem motivos para desacreditar no modelo. A análise gráfica também permite a verificação desta condição. Outra maneira de medir este efeito é pelo fator de inflação de variância (VIF), quanto maior o fator, mais forte será a multicolinearidade. Geralmente valores de VIF acima de 10 causarão problemas na estimativa dos parâmetros do modelo (MONTGOMERY; RUNGER, 2012).

\section{PROCEDIMENTOS METODOLÓGICOS}

As pesquisas do tipo "empírico-analíticas" utilizam tratamento de informação quantitativa e técnicas de registro. Segundo Gamboa (2012, p. 85), "essas informações são recolhidas por meio de instrumentos estruturados, permitindo o tratamento estatístico e a apresentação dos resultados através de esquema cartesianos, gráficos estatísticos ou quadros de correlações". Classificado como uma pesquisa científica do tipo empírico-analítica, apoiada em métodos quantitativos estatísticos, o presente trabalho de pesquisa foi direcionado a partir do seguinte questionamento: (i) Levando-se em consideração os dados de custos e de produção, é possível identificar algum tipo de correlação entre "custos totais de produção" e os dados de produtividade, mortalidade, consumo de ração e horas trabalhadas na produção de ovos? (ii) Se identificado um grau de correlação satisfatório entre as variáveis em estudo, é possível conceber um modelo matemático capaz de linearizar o comportamento de "custos totais" associados à produção de ovos, no estado do Paraná?

Para responder aos questionamentos propostos nesta pesquisa, inicialmente, foi realizada a revisão bibliográfica sobre custos e sobre a metodologia estatística de regressão linear múltipla, considerando sempre a sua aplicabilidade no processo análise e estimativa de custos.

Para realização desta pesquisa, foram considerados os dados do custo mensal (fixos + variáveis) da produção de ovos, referentes ao período compreendido entre março de 2016 a janeiro de 2017, de um aviário manual de produção de ovos, 
localizado na região oeste do estado do Paraná. Sendo que, a partir dos dados que compõem a amostra de pesquisa, foram identificadas também as quantidades produzidas, o consumo de ração das poedeiras, a mortalidade $\mathrm{e}$ as horas trabalhadas do processo produtivo.

A Tabela 1 mostra o detalhamento dos dados da produção de ovos, onde: o custo total de produção mensal como a variável de estudo $(\mathrm{Y})$, e a mortalidade (variável X1), produtividade (variável X2), consumo de ração (variável X3) e horas trabalhadas (variável X4), como variáveis explicativas.

Tabela 1 - Detalhamento dos dados da produção de ovos

\begin{tabular}{cccccc}
\hline Mês & $\begin{array}{c}\text { Custos } \\
\text { totais }^{\mathbf{1}}\end{array}$ & $\begin{array}{c}\text { Mortalidade } \\
\text { (galinhas) }\end{array}$ & $\begin{array}{c}\text { Produtividade } \\
\text { (Dz) }\end{array}$ & $\begin{array}{c}\text { Consumo de ração } \\
\text { (Kg) }\end{array}$ & $\begin{array}{c}\text { Horas trabalhadas } \\
\text { (mês) }\end{array}$ \\
\hline Mar. & 0,472671 & 5431 & 69,633 & 13744 & 180 \\
Abr. & 0,483926 & 5416 & 858,599 & 14073 & 165 \\
Mai. & 0,490153 & 5401 & 1238,101 & 21999 & 150 \\
Jun. & 0,489957 & 5393 & 1243,141 & 13032 & 159 \\
Jul. & 0,495981 & 5371 & 1269,908 & 21669 & 183 \\
Ago. & 0,491114 & 5360 & 1263,541 & 21027 & 186 \\
Set. & 0,49667 & 5336 & 1372,493 & 22008 & 162 \\
Out. & 0,497176 & 5320 & 1226,276 & 14012 & 135 \\
Nov. & 0,498196 & 5288 & 1075,458 & 24798 & 180 \\
Dez. & 0,499203 & 5236 & 1173,133 & 18974 & 165 \\
Jan. & 0,50095 & 5214 & 1143,666 & 18043 & 156 \\
\hline
\end{tabular}

(1) Custos totais de produção por galinha poedeira

Fonte: Elaboração própria (2017)

Após a identificação dos dados da produção, foi aplicada a metodologia estatística de análise de regressão linear múltipla para verificação do grau de relacionamento entre as variáveis estudadas e, ainda, para a identificação da equação capaz de linearizar o comportamento dos "custos totais" em função do comportamento das variáveis independentes.

Para validação das modelagens pesquisadas, além das análises envolvendo o coeficiente de determinação $\left(R^{2}\right)$, a estatística $t$ e testes de significância de coeficientes, foram realizados testes estatísticos envolvendo a análise de presença de multicolinearidade (estatísticas VIF (Variance Inflation Factor) e Tolerância (Tolerance)), autocorrelação de resíduos (estatística $d$ de Dubin-Watson) e a normalidade de resíduos (Kolmogorov-Smirnov). A combinação das variáveis se deu 
através do método de busca sequencial Backward e o nível de significância 5\%.

Observa-se que a aplicação de método estatístico relativo à análise de regressão linear múltipla requer a utilização de um programa que proporcione precisão e agilidade no processo de compilação, análise e validação dos dados da amostra escolhida. Para tanto, o programa utilizado para esta finalidade foi o STATISTICA Release 8, versão 8.0.360.0.

\section{RESULTADOS E DISCUSSÕES}

O processo utilizado no aviário em estudo é o processo manual, por ser o mais simples e o mais trabalhoso, é o que demanda um maior volume de custos relacionados à de mão de obra. Conforme já discutido na revisão bibliográfica, o processo de regressão linear múltipla permite a inclusão de mais de uma variável explicativa do comportamento dos custos.

Depois de realizado analisado todos os dados (Tabela 1), em seguida testouse a equação de regressão linear múltipla, as combinações das variáveis se deram por meio do método de busca sequencial backward. Os resultados obtidos no modelo possibilitaram a seguinte modelagem: "Y = F+V1X1+V2X2", cujo coeficiente de intercepto $(F)$ é igual 0.831507 e os coeficientes angulares V1 e V2 são 0,000066 e 0,000014, respectivamente, conforme resumido na Tabela 2.

Tabela 2 - Resumo dos coeficientes encontrados no processo de regressão linear múltipla

\begin{tabular}{lc}
\hline Coeficientes da equação & Valores e equação \\
\hline Intercepto de $Y$ & 0.831507 \\
Variável $X_{1}$ & 0,000066 \\
Variável $X_{2}$ & 0,000014 \\
\hline Equação pesquisada & $\mathrm{Y}=0.831507-0,000066 \mathrm{X}_{1}+0,000014 \mathrm{X}_{2}$
\end{tabular}

Fonte: Elaboração própria (2017).

A regressão linear múltipla apresentou um coeficiente de determinação de 0,95006292, indicando que as variáveis "produtividade" e "mortalidade" explicam 95\% das variações nos "custos totais de produção" mensais (Tabela 3). Este coeficiente indica um alto grau de relacionamento entre a variável de estudo e as variáveis explicativas. Diante disso, pode-se afirmar que os "custos totais de 
produção" mensais são altamente influenciados pela quantidade de ovos produzida e pela mortalidade das galinhas poedeiras no processo produtivo.

Diante desse resultado, pode-se considerar respondido 0 primeiro questionamento desta pesquisa, pois, tanto o coeficiente de correlação ( $R$ múltiplo) quanto o coeficiente de determinação (R-Quadrado) indicaram a existência de alto índice de relacionamento entre as variáveis "custos totais" e a "produtividade" e "mortalidade" na produção de ovos no estado do Paraná.

Tabela 3 - Resumo das estatísticas do processo de regressão linear múltipla

\begin{tabular}{lc}
\hline Tipo de estatística & Valores \\
\hline R-Quadrado ajustado & 0,950062 \\
R múltiplo & 0,979821 \\
Estatística t para teste de significância do intercepto & 17.353316 \\
Estatística t para teste de significância do coeficiente & $X_{1}-7.508330$ \\
Estatística t para teste de significância do coeficiente & $X_{2} 7.752779$ \\
Estatística F & 96.125991 \\
p-level & 0,000000 \\
p-level coeficiente $X_{1}$ & 0,000069 \\
p-level coeficiente $X_{2}$ & 0,000055
\end{tabular}

Fonte: Elaboração própria (2017).

Ainda com base nas informações contidas na Tabela 3, os testes $t$ de significância dos parâmetros estimados (intercepto e coeficientes $\mathrm{X}_{1}$ e $\mathrm{X}_{2}$ ) rejeitaram a hipótese nula, logo, conclui-se que todos os coeficientes estimados são estatisticamente significantes ao nível de $5 \%$ de probabilidade de erro. $O$ teste $\mathrm{F}$ confirma também a rejeição da hipótese de efeito nulo das variáveis "produtividade" e "mortalidade" sobre a variável de estudo "custos totais". Através dos dois testes de regressão (teste $\mathrm{F}$ e $0 \mathrm{p}$ de significância), pode-se concluir, com risco de $5 \%$ de significância que há regressão, pois o valor de $\mathrm{F}$ é maior que o tabelado e $0 \mathrm{p}$ calculado é inferior a 0,05. Logo, a hipótese $\mathrm{H}_{0}$ é rejeitada. A Tabela 4 representa o teste da normalidade dos resíduos.

Tabela 4 - Teste da normalidade dos resíduos

\begin{tabular}{l|l|l|l|l|l|l}
\hline \multirow{2}{*}{ Variável } & \multicolumn{7}{|c}{ Test of Normality (Custos) } \\
\cline { 2 - 8 } & $\mathrm{N}$ & $\max \mathrm{D}$ & $\begin{array}{l}\mathrm{K}-\mathrm{S} \\
\mathrm{p}\end{array}$ & $\begin{array}{l}\text { Lilliefors } \\
\mathrm{p}\end{array}$ & $\mathrm{W}$ & $\mathrm{p}$ \\
\hline Resíduos & 11 & 0.232435 & $\mathrm{p}>.20$ & $\mathrm{p}<.10$ & 0.939773 & 0.517833 \\
\hline
\end{tabular}

Fonte: Elaboração própria (2017). 
Na verificação da normalidade dos resíduos, segundo o teste de KolmogorovSmirnov (Tabela 4), as variáveis independentes (produtividade e mortalidade) e a variável dependente (custos totais) se mostraram com valores da probabilidade de significância ( $p$-valor) acima de 0,05 , indicando que todas podem ser descritas por distribuições normais.

Outro parâmetro observado em relação aos resíduos foi à verificação da autocorrelação, através do teste Durbin-Watson, que se baseia em cálculo de medida conhecida como Estatística DW, tabelada para valores críticos (segundo o nível de confiança escolhido) (CORRAR et al., 2007). Foi formulada a seguinte hipótese:

$H_{0}$ : Não existe correlação serial dos resíduos;

$\mathrm{H}_{1}$ : Existe correlação serial dos resíduos.

$O$ valor encontrado para o teste foi de 2,671. Como o valor deu acima do numeral 2, não sabemos se esse valor esta dentro da região considerada ausência de autocorrelação. Para isto, foi preciso considerar o tamanho da amostra (n) e o número de variáveis independentes $(p)$ para estabelecer o valor crítico inferior $\left(d_{L}\right)$ e o valor crítico superior (du). A análise se baseia na seguinte regra para comparação apresentada por Corrar et al. (2007) para os valores encontrados na estatística de Durbin-Watson $(2,671)$.

Entre 0 e dL - Autocorrelação positiva;

Entre dL e du - Não conclusivo;

Entre du e 4-du - Ausência de autocorrelação;

Entre 4-du e 4-dL - Não conclusivo;

Entre dL e 4 - Autocorrelação negativa.

Para uma amostra de 11 elementos e 2 variáveis independentes (produtividade e mortalidade), tem-se os valores de $d_{L}=0,519$ e $d u=1,297$, conforme tabela apresentada por Gujarati (2006). Logo, 4-du=2,703 e 4-dL =3,481. A Figura 1 mostra os valores críticos e a área de não rejeição da hipótese nula. 
Figura 1 - Valores críticos e a área de não rejeição da hipótese

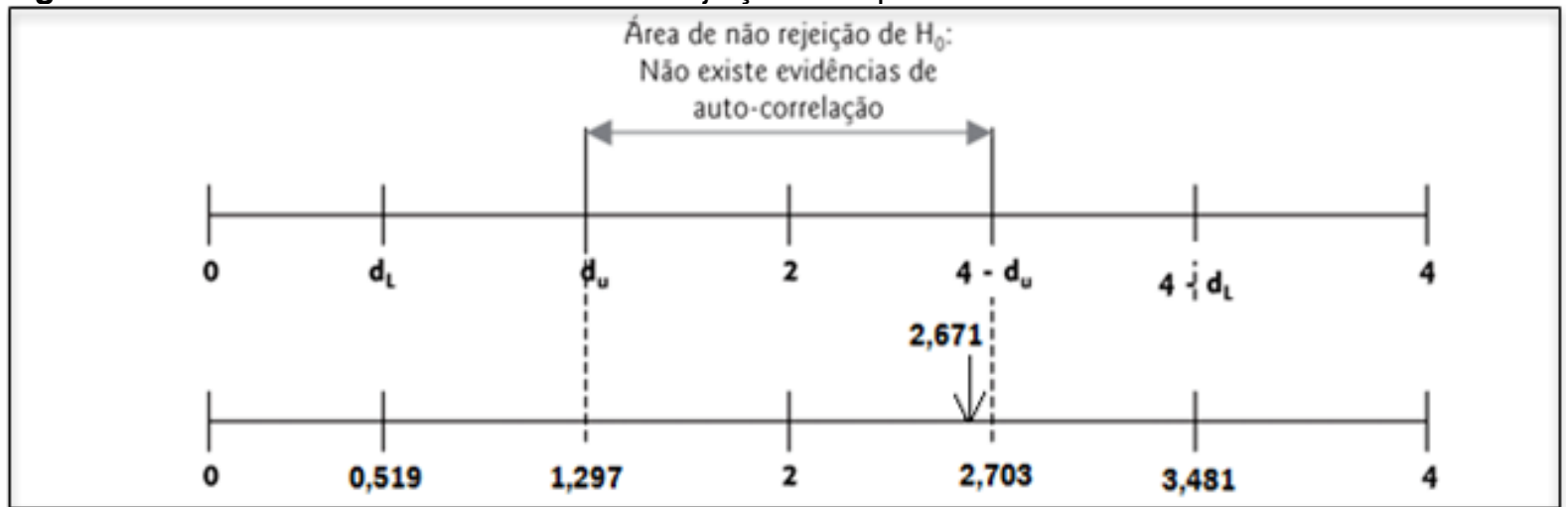

Fonte: Elaboração própria (2017)

Através da Figura 1, foi possível concluir que a Estatística DW apresentada se encontra entre du e 4-du, caracterizando ausência de autocorrelação serial, atendendo ao pressuposto da regressão.

O resultado encontrado corrobora com um estudo realizado por Castro e Martinez (2009), que ao analisar o efeito do income smoothing no mercado de ações brasileiro, com a intenção de verificar a associação entre o grau de income smoothing e seu efeito na estrutura de capital e no custo de capital de terceiros, encontraram no teste de Durbin-Watson também valores acima de dois. Os resultados rejeitaram a hipótese de autocorrelação dos resíduos, concluindo que, os estimadores podem ser considerados como melhor estimador linear não enviesado, resultados que confirmaram a robustez dos modelos utilizados.

A Tabela 5 apresentada a seguir, informa os resultados das estatísticas VIF e de Tolerância, ambas utilizadas para 0 diagnóstico da existência de multicolinearidade entre as variáveis explicativas (produtividade e mortalidade).

Tabela 5 - Resumo das estatísticas VIF e Tolerância

\begin{tabular}{lcc} 
Variável & VIF( Variance Inflation Factor) & Tolerância \\
\hline Produtividade (X1) & 1,184 & 0,844596 \\
Mortalidade (X2) & 1,184 & 0,844596
\end{tabular}

Fonte: Elaboração própria (2017)

De acordo com Fávero et al. (2009) quanto menor a estatística VIF e maior a estatística de tolerância, maior será a independência das variáveis explicativas e, por consequência, isto indicará a inexistência de multicolinearidade. Por outro lado, 
quanto maior a estatística VIF menor será a independência das variáveis explicativas e, portanto, maior a correlação entre elas, denotando, assim, fortes indícios de existência de multicolinearidade entre essas variáveis. Ainda segundo Fávero et al. (2009), uma estatística VIF igual ou maior que 5, o que representa uma tolerância de 0,20 ou menos, já é suficiente para indicar a existência de multicolinearidade entre as variáveis explicativas do modelo proposto.

Diante das informações contidas na Tabela 5, as variáveis explicativas "produtividade" e "mortalidade" não detectaram por esses dois testes problemas de multicolinearidade.

Carmo et al. (2012), propuseram um estudo de natureza empírico-analítica, a partir da variável dependente (gastos financeiros), e das variáveis independentes (montantes de custos fixos totais e montantes de custos variáveis totais) da produção de frangos para abate, em sete estados brasileiros. Por intermédio da análise de regressão linear múltipla, encontraram como resultados das estatísticas VIF e de Tolerância, que apenas dois estados brasileiros não apresentaram indícios de multicolinearidade nos modelos propostos, dois estados não podem ser considerados válidos, pois, eles apresentaram fortes indícios de existência de multicolinearidade entre as variáveis explicativas e os outros três estados podem apresentar alguns indícios de multicolinearidade, isto é, nestes três casos, os índices encontrados ainda não inviabilizam as variáveis explicativas dos respectivos modelos, contudo, merecem um pouco mais de atenção. As estatísticas d referentes ao teste de Durbin-Watson, constaram que todos os modelos pesquisados apresentaram autocorrelação dos resíduos, exceto um estado, sendo este já desqualificado anteriormente por apresentar problemas multicedasticidade e heterocedasticidade. Diante das evidências constatadas inicialmente, perceberam que seis dos sete estados pesquisados apresentarem-se aparentemente satisfatórios. Contudo, o aprofundamento do processo de análise para validação das percepções iniciais, próprio dos trabalhos de natureza científica, permitiu concluir que nenhuma das modelagens pesquisadas pôde ser considerada válida, de acordo o objetivo proposto pela pesquisa. 


\section{CONCLUSÃO}

Uma boa gestão de custos pode representar um diferencial competitivo para o produtor, uma vez que sem o gerenciamento e controle torna-se difícil atingir bons resultados, assim como, identificar se o produtor está obtendo o retorno desejado com a atividade. Além disso, com uma boa avaliação dos custos, há a possibilidade de aumento da eficiência e qualidade produtiva.

Neste trabalho, foi proposta uma metodologia baseada no modelo de regressão linear múltipla, para descrever os padrões de comportamento dos "custos totais", em função das variáveis independentes ("produtividade", "consumo de ração", "mortalidade" e "horas trabalhadas"). O método utilizado para seleção das variáveis do modelo foi o de busca sequencial backward.

Respondendo aos questionamentos propostos na metodologia, conclui-se que, houve algum tipo de correlação e foi possível determinar uma equação matemática para os padrões de comportamento dos custos totais, em função das variáveis independentes "produtividade" e "mortalidade", ou seja, através do coeficiente de determinação, observou-se que, essas duas variáveis explicaram 95\% das variações nos "custos totais de produção". As variáveis "consumo de ração" e "horas trabalhadas" não influenciaram nas variações dos "custos totais", sendo excluídas do modelo.

Quanto ao atendimento aos pressupostos, todos os pontos analisados obtiveram resultados satisfatórios em relação ao seu atendimento, sendo que foram testados os seguintes pressupostos: multicolinearidade, ausência de autocorrelação serial nos resíduos e normalidade dos resíduos, o pressuposto de linearidade dos coeficientes também foi atendido.

De acordo com os resultados dos testes estatísticos, percebeu-se que o modelo pesquisado apresentou-se satisfatório e permitiu concluir que a modelagem pesquisada pôde ser considerada válida, de acordo com o objetivo proposto da pesquisa. Recomenda-se para estudos futuros a continuidade desse estudo com mais dados a serem inseridos e com dados de outros anos. Recomenda-se, também, a utilização de dados de aviários totalmente automatizados, pois possui um custo maior de instalação. 


\section{REFERÊNCIAS}

ANDERSON, D. R. et al. Estatística aplicada à administração e economia. Tradução: José Carlos Barbosa dos Santos. 2. ed. São Paulo: Cengage Learning, 2007.

ANDERSON, D. R. et al. Estatística aplicada à administração e economia. 2. ed. São Paulo: Cengage Learning, 2011.

ANDRADE, M. G. F. et al. Controle de custos na agricultura: um estudo sobre a rentabilidade na cultura da soja. Custos e @gronegócio on line, v. 8, n. 3, Jul./Set. 2012.

AVISITE. Consumo de ovos favorece a avicultura de postura em 2016. Mundo agro. Campinas, SP : Editora Ltda, n. 40, jan. 2017.

AZEVEDO, D. B. et al. Estratégia competitiva de uma multinacional estrangeira na avicultura de postura no Brasil. Revista de Administração da UFSM, v. 5, n. 3, p. 479-492, 2012. https://doi.org/10.5902/198346596216

BIASI, A. et al. Análise do custo de produção de ovos e a oscilação no preço de venda: uma visão gerencial. PUBVET (Publicações em Medicina Veterinária e Zootecnia), Londrina, v. 5, n. 15, ed. 162, art. 1093, 2011. https://doi.org/10.22256/pubvet.v5n15.1093

BORNIA, A.C. Análise gerencial de custos: aplicação em empresas modernas. 3. ed. São Paulo: Atlas, 2010.

BRUNI, A. L.; FAMÁ, R. Gestão de custos e formação de preços: com aplicações na calculadora HP 12C e Excel. 6. ed. São Paulo: Atlas, 2012.

CARMO, C.R.S. et al. Planejamento de gastos financeiros na avicultura de sete estados brasileiros: um estudo empírico baseado na análise de regressão linear múltipla e no comportamento dos custos de produção. Custos e @gronegócio on line, v. 8, n. 1, jan./mar. 2012.

CARVALHO, F. M.; FIÚZA, M.A.; LOPES, M.A. Determinação de custos como ação de competitividade: estudo de um caso na avicultura de corte. Ciênc. agrotec., Lavras, v. 32, n. 3, p. 908-913, maio/jun., 2008. https://doi.org/10.1590/S1413-70542008000300031

CASTRO, M. A. R.; MARTINEZ, A. L. Income smoothing, custo de capital de terceiros e estrutura de capital no brasil. Revista de Administração Mackenzie, São Paulo, SP, v. 10, n. 6, nov./dez. 2009. https://doi.org/10.1590/S1678-69712009000600004

CREPALDI, S. A. Contabilidade rural: uma abordagem decisional. 5. ed. São Paulo: Atlas. 2009.

CREPALDI, S. A. Curso básico de contabilidade de custos. 5. ed. São Paulo: Atlas, 2010.

CORRAR, L. J.; PAULO, E.; DIAS FILHO, J. M. Análise multivariada para os cursos de administração, ciências contábeis e economia. São Paulo: Editora Atlas, 2007.

CORRAR, L. J.; PAULO, E.; DIAS FILHO, J. M. Análise multivariada para os cursos de administração, ciências contábeis e economia. 1 ed. São Paulo: Atlas, 2009. 
CORRAR, L. J.; PAULO, E.; DIAS FILHO, J. M. Análise multivariada para os cursos de administração, ciências contábeis e economia. 1. ed. São Paulo: Atlas; 2012.

DOWNING, D.; CLARK, J. Estatística aplicada. 2.ed. São Paulo: Saraiva, 2006.

FÁVERO, L. P. et al. Análise de dados: modelagem multivariada para tomada de decisões. Rio de Janeiro: Elsevier, 2009.

GALLO, B. Avicultura de alta performance no século 21. Disponível em: http://www.avisite.com.br/noticias/imprimir.php?codnoticia=15798. Acesso em: 21/03/2017.

GAMBOA, S.S. Pesquisa em educação: métodos e epistemologias. 2. ed. Chapecó, SC: Argos, 2012. https://doi.org/10.18675/2177-580X.vol2.n1.p09-32

GARRISON, R. H.; NOREEN, E. W. Contabilidade gerencial. Rio de Janeiro: LTC, 2001.

GUJARATI, D. N.. Econometria básica. Tradução Maria José Cyhlar Monteiro. Rio de Janeiro: Editora Elsevier, 2006.

HANSEN, D.R; MOWEN, M. M. Gestão de custos: contabilidade e controle. São Paulo: Pioneira Thomson Learning, 2003.

HELENCO, R., RIGON, C.M. Viabilidade econômica para implantação de um aviário para produção de ovos de galinha. 2015. $149 \mathrm{f}$. Trabalho de Conclusão de Curso (Graduação) - Curso Superior em Administração da Universidade Regional do Noroeste do Rio Grande do Sul, 2015.

HORNGREN, C. T.; FOSTER, G.; DATAR, S. M. Contabilidade de custos. Rio de Janeiro: LTC, 2000.

INSTITUTO BRASILEIRO DE GEOGRAFIA E ESTATÍSTICA. Estatística da produção pecuária. 2017. Disponível em:

ftp://ftp.ibge.gov.br/Producao Pecuaria/Fasciculo Indicadores IBGE/abate-leite-couroovos 201604caderno.pdf. Acesso em: 20 abr. 2017.

IUDÍCIBUS, Sérgio. Análise de custos. São Paulo: Atlas, 1989.

LEONE, G. S. G. Custos: planejamento, implantação e controle. São Paulo: Atlas, 2000.

LEONE, G. S. G.; LEONE, R. J. G. Curso de contabilidade de custos. 4. ed. São Paulo: Atlas, 2010.

MAHER, M. Contabilidade de custos: criando valor para a administração. São Paulo: Atlas, 2001.

MARTINS, E. Contabilidade de custos. 10. ed. São Paulo: Atlas S.A, 2010.

MEDEIROS, O. de. Testes Empíricos sobre o Comportamento Assimétrico dos Custos nas Empresas Brasileiras. Revista de Contabilidade \& Finanças, USP, 38., maio/ago. 2005. https://doi.org/10.1590/S1519-70772005000200005 
MEGLIORINI, E. Custos: análise e gestão. 3. ed. São Paulo: Person Prentice Hall, 2012.

MELO, A. S. Análise de custos na gestão rural: um estudo acerca do comportamento dos custos de produção de frango em Pernambuco. Custos e @gronegócio on line, v. 12, n. 1, jan/mar. 2016.

MONTGOMERY, D.C.; RUNGER, G.C. Estatística aplicada e probabilidade para engenheiros. 5. ed. LTC. 2012.

POULTRY TRENDS. Egg production breaks record. Disponível em:

http://www.poultrytrends.com/2016/index.php\#/32. Acesso em: 22/12/2016.

RAÍCES, C. Guia valor econômico de agronegócios. São Paulo. Ed.: Globo. 2003.

SANTOS, J. J. Contabilidade e análise de custos: modelo contábil, métodos de depreciação, ABC-Custeio Baseado em Atividades, analise atualizada de encargos sociais sobre salários, custos de tributos sobre compras e vendas. 6. ed. São Paulo: Atlas, 2011.

SELL, I. Utilização da regressão linear como ferramenta de decisão na gestão de custos. In: CONGRESSO BRASILEIRO DE CUSTOS, 12., 2005. Florianópolis. [Anais...] Florianópolis: 2005.

SILVA, F. D. C. S. et al. Comportamento dos custos: uma investigação empírica acerca dos conceitos econométricos sobre a teoria tradicional da contabilidade de custos. Rev. contab. finanç., v.18, n.43, São Paulo, jan./apr.2007. https://doi.org/10.1590/S151970772007000100006

SILVA, R. N. S.; LINS, L. S. Gestão de custos: contabilidade, controle e análise. 2. ed. São Paulo: Atlas, 2013.

VIEIRA, M.F.A. Efeitos de duas condições climáticas, duas linhagens e dois sistemas de ventilação no desempenho produtivo de galinhas poedeiras alojadas em sistemas verticais de criação. 2015. Tese (Doutorado em Doctor Scientiae) - Programa de PósGraduação em Engenharia Agrícola, Universidade Federal de Viçosa, 2009.

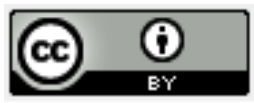

Artigo recebido em: 03/05/2018 e aceito para publicação em: 04/03/2020

DOI: http://dx.doi.org/10.14488/1676-1901.v20i1.3242 